\title{
Olivier Soutet (ed.), Jeunesse du guillaumisme
}

\section{Ruggero Druetta}

\section{Q OpenEdition}

\section{Journals}

\section{Édition électronique}

URL : http://journals.openedition.org/studifrancesi/30963

DOI : 10.4000/studifrancesi.30963

ISSN : 2427-5856

Éditeur

Rosenberg \& Sellier

\section{Édition imprimée}

Date de publication : 1 avril 2006

Pagination : 207-208

ISSN : 0039-2944

\section{Référence électronique}

Ruggero Druetta, «Olivier Soutet (ed.), Jeunesse du guillaumisme», Studi Francesi [En ligne], 148 (XLX |

I) | 2006, mis en ligne le 30 novembre 2015, consulté le 22 avril 2021. URL : http://

journals.openedition.org/studifrancesi/30963; DOI : https://doi.org/10.4000/studifrancesi.30963

Ce document a été généré automatiquement le 22 avril 2021.

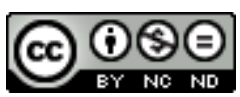

Studi Francesi è distribuita con Licenza Creative Commons Attribuzione - Non commerciale - Non opere derivate 4.0 Internazionale. 


\title{
Olivier Soutet (ed.), Jeunesse du guillaumisme
}

\author{
Ruggero Druetta
}

\section{RÉFÉRENCE}

OLIVIER SOUTET (ed.), Jeunesse du guillaumisme, « Le Français moderne », 2003, vol. 71, n.1.

1 Ce numéro de la revue Le Français moderne, coordonné par Olivier Soutet, fait état de la «jeunesse » de la théorie psychomécanique en ce qu'il rassemble des contributions de jeunes chercheurs ayant soutenu, dans les trois années précédentes, des thèses s'appuyant sur des analyses d'inspiration guillaumienne. La richesse de ces contributions est un signe de la fécondité d'une théorie complexe, qu'on ne peut réduire à quelques principes et hypothèses généraux, mais qui peut encore constituer matière à débat.

2 MATHIEU VALETTE, Énonciation et cognition: deux termes in absentia pour des notions omniprésentes dans l'oeuvre de Guillaume (pp. 6-25).

3 L'A. analyse les aspects épistémologiques de la pensée de Guillaume, qu'il éclaire à l'aide notamment de nombreux fragments manuscrits inédits. L'exégèse d'une construction textuelle des termes et des concepts telle que la pratiquait Guillaume doit se mesurer avec des définitions qui s'élaborent en se disant et dont les facettes ont parfois du mal à s'harmoniser. Les trois points analysés dans cet article concernent avant tout le rapport entre pensée et langage, avec le risque d'un point de vue psychologique que Guillaume rejetait. Un deuxième point concerne la signification exacte du terme "psychomécanique », par lequel Guillaume désignait l'aspect à la fois déterminé et intentionnel du mécanisme téléologique qu'est la langue. Un dernier point est constitué par le sujet du langage: Guillaume est convaincu qu'il faut retrancher tout ce qui est subjectif de la théorie de la langue pour asseoir son caractère scientifique : le seul sujet possible, dans une théorie scientifique de la langue, sera alors 
le sujet externe observant le mécanisme, à savoir le linguiste lui-même, et non le locuteur (sujet interne), qui n'a pas conscience des opérations langagières.

Philippe MONNERET, Les exigences théoriques d'une neurolinguistique guillaumienne (pp. 26-36).

5 L'A. propose une tentative de validation de la théorie psychomécanique par son application aux troubles de langage et ébauche ce que pourrait être une neurolinguistique guillaumienne. Si les tentatives de validation ont presque toujours échoué, c'est que, d'une part, les guillaumiens ne sont toujours pas d'accord sur le caractère réel du temps opératif et que, d'autre part, les neurosciences n'admettent pas volontiers de théorie linguistique indépendante de l'observation des troubles de langage, la seule exception étant celle de la grammaire générative, dont la validation neuropsychologique ne fait pourtant pas l'unanimité. En dépit de ces conditions défavorables, l'A. essaie de donner une interprétation psychomécanique à l'agrammatisme de Broca, en inscrivant dans un schéma d'ordination opérative (saisies précoce et tardive) les couples autour desquels se distribuent les troubles (prédicatif/ non prédicatif; auxiliaire/non auxiliaire; verbe/nom; consonne/voyelle etc.), l'ordonnancement reposant sur les notions de subduction, d'incidence et de fréquence. Il en résulte que les éléments altérés dans ce type d'aphasie sont toujours des éléments d'avant. Cette perspective permettrait d'éclairer le caractère variable de ces troubles, car les tensions opératoires sont des processus de nature continue, et d'échapper à l'alternative entre une explication structurale et procédurale, car le processus est ici inscrit dans la compétence.

STÉPHANIE THAVAUD-PITON, Guillaume, lecteur de Bréal (pp. 37-51).

7 L'A. se penche sur le rapport entre Guillaume et Bréal, que l'inventeur de la psychomécanique cite à plusieurs reprises dans ses travaux. Les mécanismes de la sémantique historique de Bréal ainsi que la relation entre les évolutions diachroniques et la compétence isosynchronique des locuteurs sont interprétés par Guillaume à la lumière de la notion de temps opératif, qui permet d'effectuer le lien entre ces deux dimensions et d'en expliquer l'autonomie relative. Les «lois» de Bréal sont par conséquent réinterprétées à la lumière de ce concept: la loi d'irradiation (transfert d'une partie de la sémantèse du radical au suffixe, qui peut le transporter à son tour à d'autres radicaux) s'explique en termes de répartition de la sémantèse sur les deux tensions du mot, ce qui la soustrait à l'action de la volonté humaine. Sont ensuite passés en revue d'autres points de la théorie de Bréal (la loi de répartition et la loi de restriction); là aussi, l'apport guillaumien va toujours dans le sens d'une autonomisation des mécanismes linguistiques vis-à-vis des facteurs sociaux et intentionnels. Le traitement de la polysémie, enfin, montre que derrière la reprise du schéma explicatif de Bréal, la pensée de Guillaume exclut en fait la permanence d'un signifié de puissance commun à tous les effets de discours, au profit d'une unité de physisme (forme) susceptible de réunir plusieurs psychismes de signifiance.

KIRILL ILINSKI, Les degrés de l'incidence (pp. 52-67).

9 L'A. rappelle les termes de la problématique de l'incidence et de son application aux parties du discours, à travers le cas du substantif, de l'adjectif et de l'adverbe. Après avoir montré que la description en termes d'incidence interne pour le substantif n'est pas exempte de critiques (du point de vue de sa définition, elle frôle la tautologie et, du point de vue syntaxique, l'incidence du substantif à l'article risque d'entrer en contradiction avec cette définition), l'A. caractérise l'incidence interne associée aux 
différents types de nom (nom propre, pronom, indéfini), puis s'attaque à l'incidence adjectivale, dont il démontre l'incidence externe du second degré (à savoir une incidence à l'incidence nom-article, et non pas une incidence du premier degré au substantif seul). L'adverbe aussi est doté d'une incidence externe du second degré (principalement au verbe), la différence principale entre adjectif et adverbe résidant dans le fait que ce dernier " passe obligatoirement par un terme d'appui qui constitue un apport au support personnel visé par l'adverbe ", alors que "l'apport de l'adjectif peut ne pas transiter sémantiquement par l'apport du substantif pour venir qualifier le référent qu'il vise » (p. 63).

Béatrice CHAPUT, La notion de prototype morphosyntaxique dans l'analyse de certains transferts catégoriels (pp. 68-78).

A partir d'un certain nombre d'exemples problématiques de transferts catégoriels (ou, dans les termes de Tesnière, de translations), recensés dans les exemples du Bon Usage, l'A. s'interroge sur le bien-fondé de la division traditionnelle des parties du discours : la possibilité de transferts catégoriels risque en effet de rendre tout à fait arbitraire ce type de classement. Les exemples analysés portent sur la dérivation morphologique (ex. aller chattement; dans le parc où brument les ennuis...), sur la dérivation au moyen d'un outil (ex. infinitif nominal : de manier la monnaie affine d'une certaine manière; infinitif de narration: Edouard de s'inquiéter) et enfin sur la dérivation sans marque morphologique et sans outil (ex. sa mère que j'avoue que je redoute fort; cette durée collective qui englobe et dépasse les nôtres individuelles). Dans tous ces cas, l'A. parvient à ramener les emplois observés en discours au spectre morphosyntaxique du signe en langue et démontre ainsi que la notion guillaumienne de régime incidentiel d'une partie de langue s'avère suffisamment souple pour autoriser des dérivations discursives plus ou moins considérables et, partant, préférable au cloisonnement des «catégories grammaticales ». PIERRE-YVES DUFEU, L'impératif du verbe ‘ aller > dans l'histoire du français (pp. 79-98).

13 L'A. analyse les occurrences de l'impératif du verbe "aller» dans un corpus diachronique, allant du XII ${ }^{e}$ au XVIII ${ }^{e}$ siècle, ce qui lui permet de ramener les différents sémantismes observés en synchronie à des constructions syntaxiques diachroniquement bien attestées. Après avoir décrit les différents types syntaxiques, que l'A. nomme processuel, locatif, circonstanciel et nucléaire, il montre comment ceux-ci s'organisent autour d'un double mouvement systématique d'orientation inverse: un mouvement d'intériorisation du contenu sémique (par un principe de projection qui détermine, à l'issue du processus, la polysémie des formes nucléaires d'impératif, qui contrastent nettement avec les emplois de l'indicatif et du subjonctif), puis un mouvement d'extériorisation morphématique. Celui-ci est particulièrement sensible dans les emplois interjectifs de l'impératif de "aller ", jusqu'aux formes lexicalisées diva $\left(\mathrm{XII}^{\mathrm{e}} \mathrm{s}\right.$.) et oui da $\left(\mathrm{XVI}^{\mathrm{e}} \mathrm{s}\right.$.). Le modèle cinétique guillaumien permet donc dans ce cas d'offrir une vision unifiée et cohérente des divers emplois et signifiés de cette forme verbale. 